\title{
Reinforcement Learning Experience Reuse with Policy Residual Representation*
}

\author{
WenJi Zhou ${ }^{1}$ and Yang Yu${ }^{1}$ and Yingfeng Chen $^{2}$ and Kai Guan ${ }^{2}$ and \\ Tangjie $\mathbf{L v}^{2}$ and Changjie Fan ${ }^{2}$ and Zhi-Hua Zhou ${ }^{1}$ \\ ${ }^{1}$ National Key Laboratory for Novel Software Technology, Nanjing University, Nanjing, China \\ ${ }^{2}$ NetEase Fuxi AI Lab, Hangzhou, China \\ \{zhouwj, yuy, zhouzh\}@lamda.nju.edu.cn, \\ \{chenyingfeng1,guankai1,hzlvtangjie,fanchangjie\}@corp.netease.com
}

\begin{abstract}
Experience reuse is key to sample-efficient reinforcement learning. One of the critical issues is how the experience is represented and stored. Previously, the experience can be stored in the forms of features, individual models, and the average model, each lying at a different granularity. However, new tasks may require experience across multiple granularities. In this paper, we propose the policy residual representation (PRR) network, which can extract and store multiple levels of experience. PRR network is trained on a set of tasks with a multi-level architecture, where a module in each level corresponds to a subset of the tasks. Therefore, the PRR network represents the experience in a spectrum-like way. When training on a new task, PRR can provide different levels of experience for accelerating the learning. We experiment with the PRR network on a set of grid world navigation tasks, locomotion tasks, and fighting tasks in a video game. The results show that the PRR network leads to better reuse of experience and thus outperforms some state-of-the-art approaches.
\end{abstract}

\section{Introduction}

Reinforcement learning [Sutton and Barto, 1998] has recently shown many impressive results. It has achieved human-level performance in a wide variety of tasks, including playing Atari games from raw pixels [Guo et al., 2014; Mnih et al., 2015; Schulman et al., 2015], playing the game of go [Silver et al., 2016] and robotic manipulation [Levine et al., 2016; Lillicrap et al., 2015]. However, most of them have very high sample complexity. For example, to master a single Atari game, state-of-the-art methods need tens of thousands of interactions. However, while facing a new task, the same quantity of samples needs to be collected again in traditional reinforcement learning methods. In contrast, human beings can

${ }^{*}$ This work is supported by the National Key R\&D Program of China (2018YFB1004300), NSFC (61751306, 61876077), Jiangsu SF (BK20170013), and Collaborative Innovation Center of Novel Software Technology and Industrialization. This work is partially done while Wen-Ji Zhou was interning at Fuxi AI Lab. Yang Yu is the corresponding author. learn a good policy through limited trials on a new task, when we have sufficient experience. It's essential to utilize knowledge learned from experienced tasks, in order to achieve efficient reinforcement learning.

Various ways have been investigated to enable reinforcement learning with the ability of experience reuse. In the process of experience reuse, the first step is how the experience is represented and stored. The experience can be stored in the forms of features, individual models, and an average model. In, e.g., [Barreto et al., 2017; BouAmmar et al., 2012], the experience is stored as the features learned from the old tasks. In, e.g., [Yu et al., 2018; Hu et al., 2018; Zhang et al., 2018], the experience is stored in individual models, each of which was trained on an old task. The reuse is to find a combination of the individual models on the new task. In, e.g., [Finn et al., 2017; Gupta et al., 2018], the experience is stored in an average model, where the average was measured as the gradient update steps to reach the optimal model over the old tasks. The reuse is to start with the model and continue the training on the new task. We notice that each previous method represents and stores the experience in a particular granularity, which does not only restrict the effectiveness of the reuse, but also make assumptions on the relationship between the new tasks and the old tasks. It could be useful for a very similar task to reuse samples directly, but for a dissimilar task, only the average model could be helpful. Therefore, for a more practical method of experience reuse, it could be beneficial that the experience is represented in multiple levels.

In this work, we propose a multi-level model architecture, named as policy residual representation (PRR), as well as the training method that enables a single model to represent multiple levels of experience. In each level of PRR, there are one or more component modules, corresponding to a subset of the tasks. The training starts from the top level with one module corresponding to all the tasks, i.e., the module is the policy that maximizes the sum of (normalized) reward on all the tasks. In other words, the top level learns the average policy over all the tasks. In each following level, a module is learned over a selected subset of tasks according to a predefined mask. Moreover, when learning the module, all the upper levels are fixed, and thus the module learns a residual policy over the selected tasks. In this way, PRR forms a multilevel architecture, where the experience of different granular- 
ities can be represented. When learning in a new task, the reuse of the PRR experience can have two phases: the first is to select the experience by learning the weights of the modules in all the levels, then a new module can be appended to learn the residual policy specific to the new task.

We test PRR with the reinforcement learning algorithm PPO [John Schulman, 2017] on three sets of environments, a set of grid-world navigation tasks, a set of Mujoco [Todorov et al., 2012] controlling tasks, and a set of fighting tasks in a video game. Experiment results not only show that PRR leads to superior performance with experience reuse to some state-of-the-art methods, but also verify the applicability of PRR to various tasks.

\section{Background}

\section{Reinforcement Learning}

Reinforcement learning aims at learning the optimal policy model from autonomous interactions with the environment. The reinforcement learning problem can be expressed as a Markov decision process. In this paper, we adopt the standard deep reinforcement learning setting. $S$ denotes the state set of the environment and $A$ denotes the action set of an agent. The agent interacts with an environment over periods of time according to a behavior policy $\pi_{\theta}$. We usually use a neural network to learn a policy $\pi_{\theta}$ and $\theta$ denotes its parameters. At each time step $t$, the agent gets a state observation $s_{t} \in S$ from environments. Then it samples an action $a_{t} \sim \pi_{\theta}\left(s_{t}\right)$ and applies the action to the environment. The environment then returns a reward $r_{t}$ sampled from an unknown reward function $R\left(s_{t}, a_{t}\right)$ to the agent and shifts to a new state $s_{t+1}$ from an unknown transition function $P\left(s_{t+1} \mid s_{t}, a_{t}\right)$. Meanwhile, the environment will notify the agent whether to terminate the current episode. The goal of agent is to maximize the expected future discounted reward $\eta\left(\pi_{\theta}\right)=E_{\tau}\left[\sum_{t=0}^{T} \gamma^{t} r_{t}\right]$, where $\tau$ denotes the whole trajectory.

However, sampling inefficiency is one of the main difficulty in reinforcement learning. For example, the state-of-the-art methods need tens of thousands of experience to learn a policy on a video game. Especially when the dimension of the state is too high or the environment is complex, the sampling efficiency of traditional reinforcement learning is very limited.

\section{Experience Reuse}

Experience reuse from old tasks is an effective way to improve the sampling efficiency of reinforcement learning on new tasks. The first step is to represent and store the experience. Some approaches [Barreto et al., 2017; Bou-Ammar et al., 2012], store the experience as the features learned from the old tasks. Some approaches [Yu et al., 2018; $\mathrm{Hu}$ et al., 2018] store the experience in individual models. Each of the individual models is trained on an old task. The experience can be reused by finding a combination of the individual models on the new task. And another kind of approaches, e.g. MAML [Finn et al., 2017] and MAESN [Gupta et al., 2018], store the experience in an average model. The average was measured as the gradient update steps to reach the optimal model from the old tasks. The reuse is to start with this average model and continue training on the new task.

However, each previous method represents and stores the experience in a particular granularity. The fixed granularity will restrict the effectiveness of the reuse and make assumptions on the relationship between the new tasks and the old tasks. These methods have been shown useful, for learning new tasks with certain types of similarity with the old tasks. However, they may fail for tasks with a different similarity type that requires a different level of experience.

Different from the existing methods, this paper presents the policy residual representation (PRR) network, which aims at extracting multiple levels of experience by learning policy residual representations with its multi-level structure.

\section{Policy Residual Representation Network 3.1 PRR Architecture}

Policy residual representation (PRR) is a multi-level neural network architecture. But unlike multi-level architectures in hierarchical reinforcement learning that are mainly used to decompose the task into subtasks, PRR employs a multi-level architecture to represent the experience in multiple granularities.

In our setting, we have a set of experience tasks $E=$ $\left\{e_{1}, e_{2}, \ldots, e_{n}\right\}$, which share the same state space and action space. A PRR model is to be trained in these environments, and stores the experience in different granularities. The overall idea of PRR is illustrated in Figure 1. Firstly, we show the structure of PRR network in Figure 1 (a). PRR is a multilayer network structure, each layer has one or more modules. $L_{i j}$ denotes the $j$-th module in the $i$-th level. Each module receives a state as input and outputs a component of the action. The outputs are all summed up according to the linear weights $\boldsymbol{w}$ to obtain the final action distribution, i.e.,

$$
\boldsymbol{a}=w_{0} \boldsymbol{a}_{0}+w_{11} \boldsymbol{a}_{11}+\ldots+w_{i j} \boldsymbol{a}_{i j} .
$$

We restrict that $\boldsymbol{w}$ is a unit vector, i.e., $\|\boldsymbol{w}\|_{1}=1$. Also, note that it is not straightforward to define what is a "negative" task, therefore, we require that each $w$ is none negative, i.e., $w_{i j} \in[0,1]$. Each module learns a representation of policy residuals. Thus this structure is called policy residuals representation network.

\subsection{Experience Acquisition with PRR Model}

In order to learn the experience from different granularity, we train the modules in a PRR sequentially, in a top-down manner. First, we need to prepare the masks, which specify the tasks selected for the modules. The multi-granularity of the experience representation has a nature similarity of the spectrum representation, thus it is natural to think of the Walsh functions [Lackey and Meltzer, 1971] to generate the masks, which achieves a spectrum transformation. However, Walsh functions generate codes with 1 's and -1 's, but it is unnatural to define a negative reinforcement learning task. For example, while "achieving the goal" can correspond to an optimal policy, "not achieving the goal" corresponds to infinite polices. Therefore, a possible modification from the Walsh functions can be that we separate 1's and -1's as different sets of tasks, 


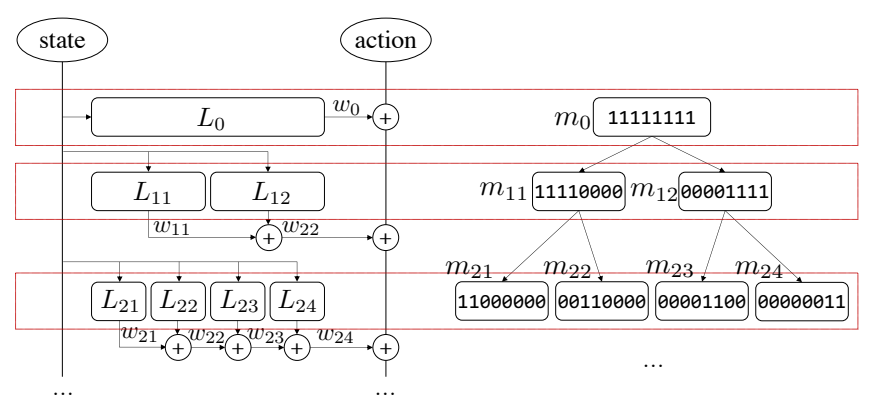

(a) PRR architecture

(b) an example of masks

Figure 1: Architecture of PRR. (a) the multi-level structure of PRR, where $L_{i j}$ denotes the $j$-th module in the $i$-th level, and the modules' output are summed up with weights $w_{i j}$ to achieve the final action (b) an example of hierarchical masks that specify the task subset for each module.

each is used to train a module. Other ways to define the masks are possible, however, in this paper we do not focus on finding the best masks. We focus on validating the idea of PRR. Therefore, we set a mask $m_{i j}$ as a binary vector, which has the same length with the number of tasks. Being 1 in the mask means the corresponding task is included for the module, and 0 means excluded.

The masks used in this paper are shown in Figure 1 (b). The mask $m_{i j}$ is a binary vector, which has the same length with the number of tasks. Each dimension corresponds to a task. $m_{i j}^{k}=1$ indicates that we select $k$-th task into training task sets for $L_{i j}$. When training $L_{i j}$, we uniformly sample a task from the selected task set and train $L_{i j}$ on this task. Then another task is uniformly sampled and $L_{i j}$ is trained. In this process, the parameters of other modules are frozen.

By learning on a subset of previous tasks, $L_{i j}$ extracts experience of a certain granularity. For example, we usually let $L_{0}$ module learn the overall experience of this series of tasks, which is the most coarse-grained experience. So its mask $m_{0}$ is a vector that all elements are 1 . If we set $m_{i j}$ to a zero-vector except for one dimension, the $L_{i j}$ will learn on a single task, by which $L_{i j}$ learns the most fine-grained experience. So we use masks to train PRR from top to bottom, from coarse-grained to fine-grained, as shown in Figure 1. This experience acquiring process is shown in 2 .

To avoid PRR network overfits a certain environment, we need to ensure that every task has the same importance. So we scale rewards on different tasks to the same range. We can apply a policy gradient based reinforcement learning approach to optimize parameters of $L_{i j}$ and $\boldsymbol{w}$. We choose to apply PPO [John Schulman, 2017] in this paper.

\subsection{Experience Reuse with PRR Model}

When we want to reuse PRR to a new task, we can first try to recombine existing modules. It means that we freeze parameters of all the modules and only train the weights of linear combination $\boldsymbol{w}$. Because there are fewer parameters to be optimized, PRR can transfer to the new task rapidly.

However, if the new task is not so much similar to previous tasks, the experience stored in PRR maybe not enough
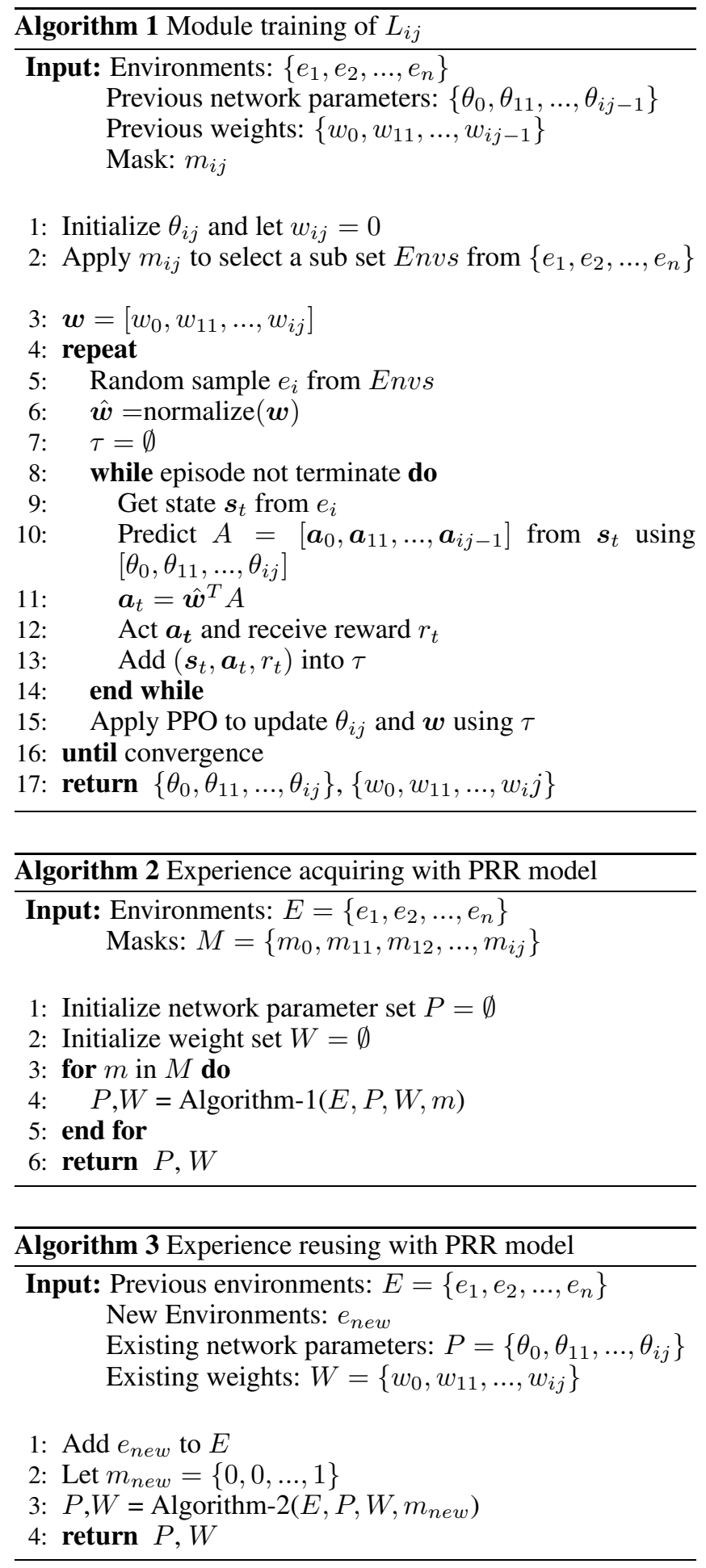

to solve it well. Thus a new module $L_{\text {new }}$ should be added to PRR and it learns policy residual representation on a new task. Because PRR has learned the coarse-grained experience of this kind of tasks, we let the mask $m_{\text {new }}=\{0,0, \ldots, 1\}$. It means that the selected task set for $L_{n e w}$ only contains the new task, which let $L_{\text {new }}$ learn the fine-grained experience on the new task. During training, our approach only updates the 
parameters of $L_{n e w}$ and $w_{n e w}$ and freezes other parameters. The learning process of transferring to a new task is shown in Algorithm 3.

\section{Experiments}

We conduct several experiments to demonstrate that PRR can learn different levels of experience from old tasks and reuse the experience to learn on a new task, by learning different levels of policy residual representation. In all these experiments, we use PPO as a basic reinforcement learning algorithm of PPR. We train PRR on level 0 and level 1 in all these experiments. On level 0 , as mentioned before, we train $L_{0}$ to learn the overall experience of this series of tasks, which is the most coarse-grained experience. On level 1, we learn the most fine-grained experience. The mask of $L_{1 j}$ is a zero vector except the $j$-th element, which means that we train $L_{1 j}$ only on $j$-th environment.

We compare our method with basic PPO [John Schulman, 2017], MLSH [Frans et al., 2018], MAML[Finn et al., 2017] and MAESN[Gupta et al., 2018]. Basic PPO is a baseline algorithm that is directly trained on a new environment. MLSH, MAML and MAESN are Meta-RL algorithms. MLSH also uses a multi-layer structure. It learns common low-level skills from a series of similar environments and reuses them on new environments. All comparison algorithms use the same hyperparameters in the PPO algorithm. The results of all experiments are the average of 10 repeated experiments.

\subsection{Complex Grid World Environment}

In order to test the performance and analyze the details of the learning process, we first validate our architecture on a complex grid world navigation tasks, which is called FetchTheKey tasks.

FetchTheKey environment is a grid world environment with many rooms. Some rooms are connected by doors and doors are initially locked. The agent can open the door only if it has the right key. The room that has the first key can be entered freely. After that, the agent needs the key $i$ to enter the room that has key $i+1$. And if the agent has all the keys, it can enter the final room to reach the goal. The positions of keys are randomly initialized every time we reset the environment, while the position of the goal is fixed. FetchTheKey a hard environment because of narrow doors, long horizon, and sparse reward. And the difficulty grows up with the increase of the number of keys. Details of the environments are described in the longer version of this paper available from http://lamda.nju.edu.cn/yuy.

\section{Overall Performance}

In this experiment, we set key $=1$ and key $=2$ environments as known tasks. We want to train PRR on these tasks and then adapt to key $=3$ environment.

As mentioned before, we first scale the total rewards of all environment to 10. PPO, as a baseline algorithm, learns on $k e y=3$ environment from scratch for 7000 episodes. MLSH sets four skills and first learns skills on key $=1$ environment and key=2 environment for 8500 episodes. And then MLSH transfer to key=3 environment for 7000 training episodes, which the same training episodes as basic PPO baseline.
MAML and MAESN also have 8500 episodes for their metatraining phase on key $=1$ and $k e y=2$ environment. And then they do meta-testing phase on key=3 environment for 7000 episodes. PRR have similar experiments setting with other approaches. It first learns $L_{0}$ on key $=1$ environment and key=2 environment for 3000 episodes. Then it learns $L_{11}$ on key $=1$ environment for 1500 training episodes and trains $L_{12}$ on key=2 environment for 4000 episodes. So PRR totally learns 8500 episodes in its training phase, which is the same as other approaches. Finally, it adopts to key $=3$ environment for 7000 training episodes and trains $L_{13}$.

The learning curves on $k e y=3$ environment are shown as Figure 3. This environment is quite difficult because of the long horizon and sparse reward. So the PPO is hard to converge and can not achieve the maximal return. Our method has a very high return at the beginning and goes up to maximal return. It indicates that PRR can extract multiple granularities of experience and select proper modules to reuse in a new task, which leads to a good beginning and better exploration.

To make a further analysis of PRR, we look into its learning process. MLSH also have multi-level architecture and we compare with it. They are both trained on key $=1$ task and $k e y=2$ task and then reuse experience to learn key=3 task. As shown in Figure 2, PRR learns $L_{0}$ to extract coarse-grained experience and achieve a good return. MLSH learns slower than PRR but it still achieves a good result at round return 9. And then they are trained on key $=1$ environment. PRR reuse the experience in $L_{0}$ to learn $L_{11}$ and the return is 10 all the time. MLSH learns fixed granularity of experience and doesn't learn new policy, it learns a new high-level policy and fine-tuning its low-level skills. Then they turn to key=2 environment. PRR still have a good beginning at return 9 and keep going up by adding a new module $L_{12}$ to fine-grained, lowlevel experience on key=2 environment. However MLSH goes up slowly, which is shown in Figure 2(c). The gap is further widened when transferring to key $=3$ environment. It shows that fixed granularity of experience can't handle new tasks that dissimilar to old tasks.

\section{Analysis of Experience Selecting}

We wonder if it's essential to add a new network for key=3 environment. So we let PRR adopt to the new environment by optimizing weights $\boldsymbol{w}$ only and analyze its performance.

In this experiment, we train $L_{0}$ on key $=1$ and key=2 environment for 3000 episodes. Then learn $L_{11}$ and $L_{12}$ on $k e y=1$ environment and key $=2$ environment respectively for 1000 episodes and 3500 episodes. And when PRR network adopts to key $=3$ environment, we don't add new networks and only train weights $\boldsymbol{w}$ to recombine $L_{0}, L_{11}$ and $L_{12}$.

We plot the learning curve of weight-only transfer, which is called PRR (weights-only) and compare to the original PRR method. The learning curves on key $=3$ environment are shown in Figure 4. PRR (weights-only) begins at a good point around 7.5. The return actually goes up by optimizing weights only. However, it grows very slow and can hardly reach the optimal return at 10 . The result indicates that there are gaps between optimal policy and learned policy residual representations. This can not be easily filled by adjusting the 


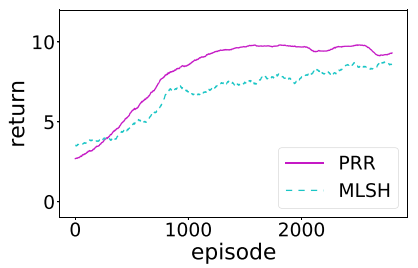

(a) Learning $L_{0}$

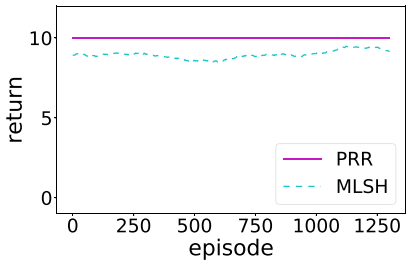

(b) Learning $L_{11}$

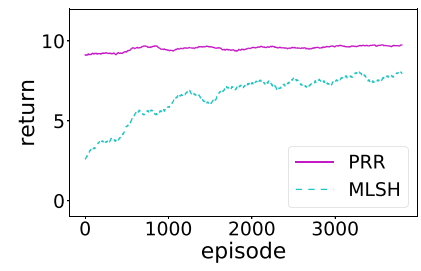

(c) Learning $L_{12}$

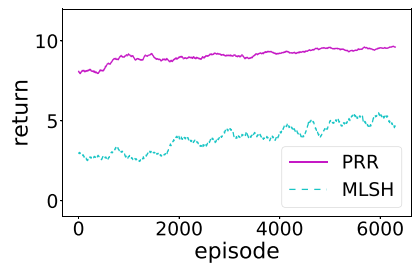

(d) Learning $L_{13}$

Figure 2: The training process of PRR and the comparison to MLSH.

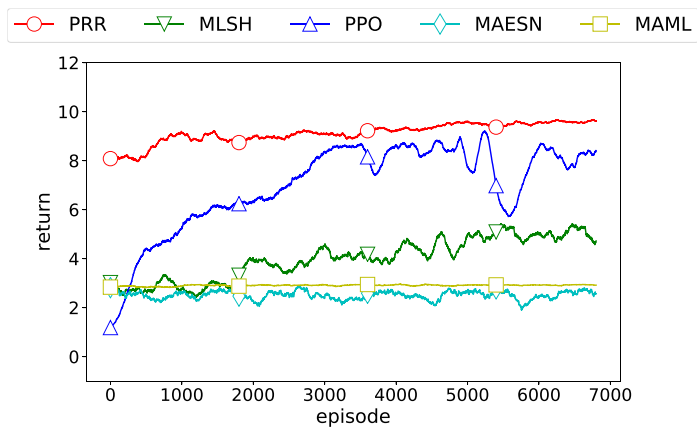

Figure 3: Learning curves on key=3 environment.

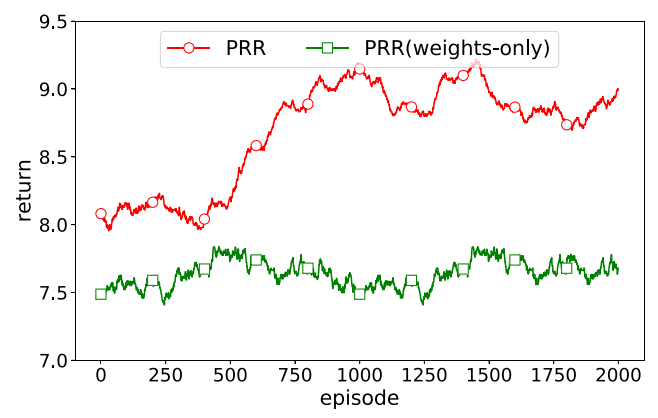

Figure 4: Learning curves on key=3 environment by PRR (weightsonly) and PRR

weights. So it's essential to learn a new policy residual for a new environment to extract more experience.

We also record and plot the change of weights $\boldsymbol{w}$ during the learning process in this experiment, in order to figure out how does PRR select experience. See Appendix for results and detailed analysis.

\section{Compare with $L_{0}$-only Learning}

Another issue to be investigated is whether the multiple levels in PPR is really helpful, or it could just the same as the single level architecture (i.e., $L_{0}$ only). In this section, we validate the necessity of the proposed residual learning architecture.

We still want to transfer from key=1 and key=2 environments to key $=3$ environment. We learn $L_{0}$ on key=1 and key=2 environments for 3000 episodes. And then transfer $L_{0}$ to key=3 environment directly by learning on this environment for 7000 episodes. This process is like course learning and we denote it as PRR ( $L_{0}$-only) methods. To compare with this method, origin PRR also learns $L_{0}$ on key=1 and key=2 environments for the same episodes. But the full PRR, moreover, learns the residuals on $k e y=1$ and $k e y=2$ environments, i.e., $L_{11}$ and $L_{12}$ and learn a new policy residual on key=3 environment.

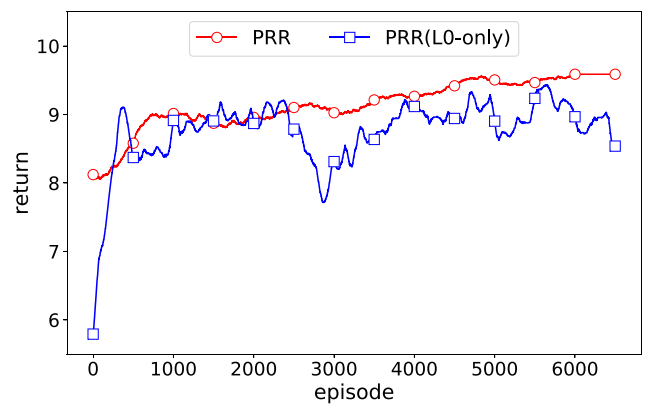

Figure 5: Transfer to key=3 environment by $\operatorname{PRR}\left(L_{0}\right.$-only $)$ and $P R R$

The result is shown in Figure 5. PRR ( $L_{0}$-only) starts at a lower point than PRR on key=3 environment. And then it closes to PRR rapidly after some beginning episodes. The rapid grow could due to that it has fewer parameters to learn. However, PRR ( $L_{0}$-only) does not converge to the performance as good as the PRR and shows an unstable learning curve.

The result shows that although a single policy network with previous experience leads to a quick learning process, it still can't capture different granularities of experience in a set of environments. PRR learns from different levels of experience leads to more comprehensive capturing of environments characteristics. And this will result in better performance. Therefore, learning different granularities of experience on different environments is essential.

\subsection{Mujoco}

We also validate our approach on robot control tasks. SwimmerGather environment is a hard robot control tasks on $\mathrm{Mu}-$ joco physics engine [Todorov et al., 2012]. The agent is a snake and moves by twisting its body. It gets a reward of 1 for collecting green balls and a reward of -1 for collecting the red ones and no other rewards.

We set three tasks, from easy to hard. For the more detailed description of these tasks, please see the Appendix. We first learn $L_{0}$ on the first task and second task for 20000 episodes. And learn $L_{11}$ on the first task for 2000 episodes and learn $L_{12}$ on the second task for 2000 episodes. Finally, 


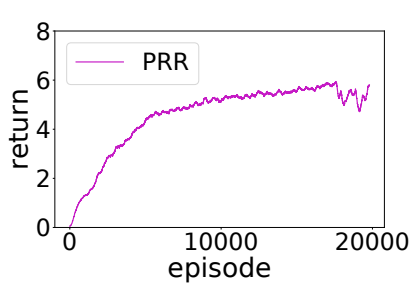

(a) Learning $L_{0}$

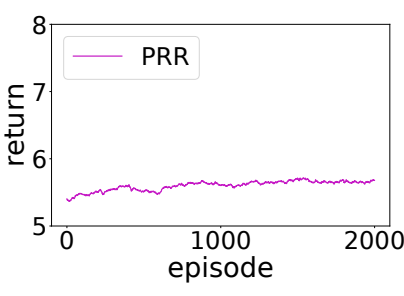

(b) Learning $L_{11}$

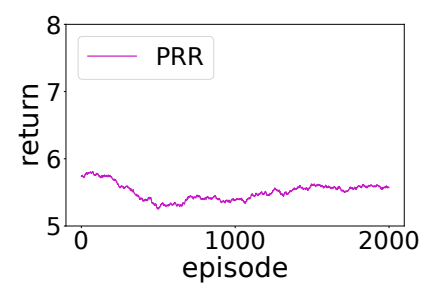

(c) Learning $L_{12}$

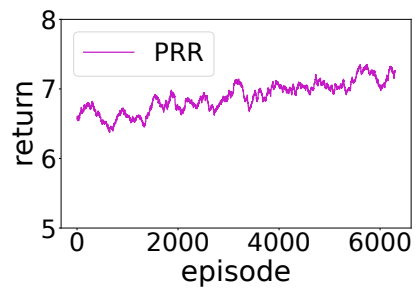

(d) Learning $L_{13}$

Figure 6: The training process on SwimmerGather environment.

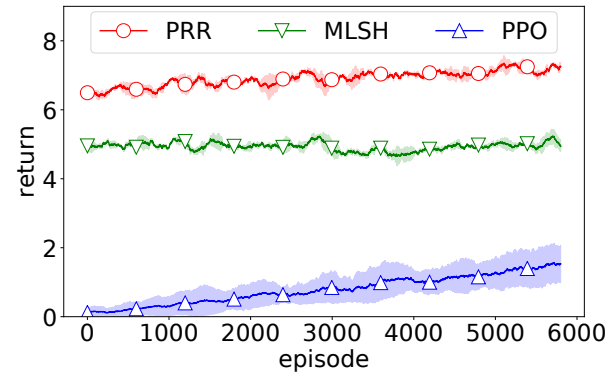

Figure 7: Learning curves on SwimmerGather environment

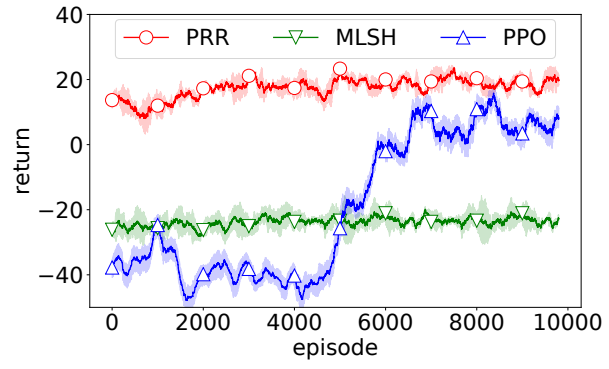

Figure 8: Learning curves on video game NiShuiHan
PRR adopts to the third environment and learns $L_{13}$. MLSH has the same number of learning episode as PRR. PPO learns on the third environment from scratch.

The learning curves are plotted in Figure 7. The PPO baseline learns very slow. On this complex environment, learning a policy from scratch is very difficult. While both MLSH and PRR reuse experience, the performance of MLSH is not as good as PRR. Due to the fixed number of skills, MLSH can hardly draw new experience on new environments. PRR learns different granularities to capture the characteristics of this kind of problems well, so it starts at a very high point in a new environment. And it learns a new module to draw new experience on the new task, which results in visible growth of return in the learning curve. The learning curves of PRR on different levels are also shown in Figure 6.

\subsection{Experiments on a Fighting Video Game}

We validate our approach on a one-on-one video game. There are many roles in this game, training models for each pair of duelists will result in huge time consumption. So we can apply PRR in this game to speed up the learning process and reuse the learned model for new roles.

In this experiment, we test on 3 roles, which are denoted as HX, TY, SM. And we train policy to control HX. So we have 3 environments, HX v.s. TY, HX v.s. SM and HX v.s. HX. We first train $L_{0}, L_{11}, L_{12}$ on $\mathrm{HX}$ v.s. TY task and $\mathrm{HX}$ v.s. SM task. And then we transfer it to HX v.s. HX game, which should be the hardest battle in this experiment. The state is extracted by humans, which is a 101-dimensional vector that contains information about the agent and its opponent. The action is a 19-dimensional vector that controls agent to move, attack and use skills. We compare PRR with PPO and MLSH in this experiment. The result is shown in Figure 8.
As shown in Figure 8, PPO need a long learning process to learn how to duel with HX. Both PRR and MLSH have a better beginning, for they both use experience learned from old tasks. However, MLSH begins at a lower point and the return almost have no raise during the training process. PRR begins at a high point, which indicates the different levels of experience can be efficiently reused on a new environment. And the return still goes up for a better result. It demonstrates that PRR can efficiently extract experience and reuse them to help training on new tasks.

\section{Conclusion}

This paper addresses the experience presentation issue for experience reuse in reinforcement learning. We propose the policy residual representation (PRR) network that consists of multiple modules organized in levels, and each module is trained on a subset of the tasks in order to extract the experience in different granularities. We also propose a training process for learning with PRR that trains the modules sequentially, in a top-down level, where a module is trained as the residual policy of the top modules. The experience reuse with a PRR model is then straightforward, by using the PRR model as the initial model with only the final level weights adjustable, leading to a quick adaptation to the new task. For the fine-grained training in the target task, a new module can also be appended to PRR, learned together with the final level weights. Our experiments on 3 sets of tasks show the effectiveness of PRR model with PPO learning algorithm. Meanwhile, we also notice that the PRR model will become huge when there are a lot of training tasks. Compact PRR models will be studied in the future approaching the goal of learnware [Zhou, 2016]. 


\section{References}

[Barreto et al., 2017] André Barreto, Will Dabney, Rémi Munos, Jonathan J. Hunt, Tom Schaul, David Silver, and Hado P. van Hasselt. Successor features for transfer in reinforcement learning. In Advances in Neural Information Processing Systems 30 (NIPS'17), pages 4058-4068, Long Beach, CA, 2017.

[Bou-Ammar et al., 2012] Haitham Bou-Ammar, Karl Tuyls, Matthew E. Taylor, Kurt Driessens, and Gerhard Weiss. Reinforcement learning transfer via sparse coding. In Proceedings of 11th International Conference on Autonomous Agents and Multiagent Systems (AAMAS'12), pages 383-390, Valencia, Spain, 2012.

[Finn et al., 2017] Chelsea Finn, Pieter Abbeel, and Sergey Levine. Model-agnostic meta-learning for fast adaptation of deep networks. In Proceedings of the 34th International Conference on Machine Learning (ICML'17), pages 1126-1135, Sydney, Australia, 2017.

[Frans et al., 2018] Kevin Frans, Jonathan Ho, Xi Chen, Pieter Abbeel, and John Schulman. Meta learning shared hierarchies. In Proceedings of the 6th International Conference on Learning Representations (ICLR'18), pages 18, Vancouver, Canada, 2018.

[Guo et al., 2014] Xiaoxiao Guo, Satinder P. Singh, Honglak Lee, Richard L. Lewis, and Xiaoshi Wang. Deep learning for real-time Atari game play using offline Monte-Carlo tree search planning. In Advances in Neural Information Processing Systems 27 (NIPS'14), pages 3338-3346, Montreal, Quebec, Canada, 2014.

[Gupta et al., 2018] Abhishek Gupta, Russell Mendonca, Yuxuan Liu, Pieter Abbeel, and Sergey Levine. Metareinforcement learning of structured exploration strategies. In Advances in Neural Information Processing Systems 31 (NIPS'18), pages 5307-5316, Montréal, Canada., 2018.

[Hu et al., 2018] Yi-Qi Hu, Yang Yu, and Zhi-Hua Zhou. Experienced optimization with reusable directional model for hyper-parameter search. In Proceedings of the TwentySeventh International Joint Conference on Artificial Intelligence (IJCAI'18), pages 2276-2282, Stockholm, Sweden, 2018.

[John Schulman, 2017] Prafulla Dhariwal Alec Radford Oleg Klimov John Schulman, Filip Wolski. Proximal policy optimization algorithms. arXiv, abs/1707.06347, 2017.

[Lackey and Meltzer, 1971] R. B. Lackey and D. Meltzer. A simplified definition of Walsh functions. IEEE Transactions on Computers, C-20:211-213, 1971.

[Levine et al., 2016] Sergey Levine, Chelsea Finn, Trevor Darrell, and Pieter Abbeel. End-to-end training of deep visuomotor policies. Journal of Machine Learning Research, 17:1-40, 2016.

[Lillicrap et al., 2015] Timothy P. Lillicrap, Jonathan J. Hunt, Alexander Pritzel, Nicolas Heess, Tom Erez, Yuval Tassa, David Silver, and Daan Wierstra. Continuous control with deep reinforcement learning. arXiv, abs/1509.02971, 2015.
[Mnih et al., 2015] Volodymyr Mnih, Koray Kavukcuoglu, David Silver, Andrei A. Rusu, Joel Veness, Marc G. Bellemare, Alex Graves, Martin A. Riedmiller, Andreas Fidjeland, Georg Ostrovski, Stig Petersen, Charles Beattie, Amir Sadik, Ioannis Antonoglou, Helen King, Dharshan Kumaran, Daan Wierstra, Shane Legg, and Demis Hassabis. Human-level control through deep reinforcement learning. Nature, 518(7540):529-533, 2015.

[Schulman et al., 2015] John Schulman, Sergey Levine, Pieter Abbeel, Michael I. Jordan, and Philipp Moritz. Trust region policy optimization. In Proceedings of the 32nd International Conference on Machine Learning (ICML'15), pages 1889-1897, Lille, France, 2015.

[Silver et al., 2016] David Silver, Aja Huang, Chris J. Maddison, Arthur Guez, Laurent Sifre, George van den Driessche, Julian Schrittwieser, Ioannis Antonoglou, Vedavyas Panneershelvam, Marc Lanctot, Sander Dieleman, Dominik Grewe, John Nham, Nal Kalchbrenner, Ilya Sutskever, Timothy P. Lillicrap, Madeleine Leach, Koray Kavukcuoglu, Thore Graepel, and Demis Hassabis. Mastering the game of Go with deep neural networks and tree search. Nature, 529(7587):484-489, 2016.

[Sutton and Barto, 1998] Richard S. Sutton and Andrew G. Barto. Reinforcement Learning - An Introduction. MIT Press, 1998.

[Todorov et al., 2012] Emanuel Todorov, Tom Erez, and Yuval Tassa. MuJoCo: A physics engine for model-based control. In Proceedings of the 2012 IEEE/RSJ International Conference on Intelligent Robots and Systems, pages 5026-5033, Vilamoura, Portugal, 2012.

[Yu et al., 2018] Yang Yu, Shi-Yong Chen, Qing Da, and Zhi-Hua Zhou. Reusable reinforcement learning via shallow trails. IEEE Transactions on Neural Networks and Learning Systems, 29(6):2204-2215, 2018.

[Zhang et al., 2018] Chao Zhang, Yang Yu, and Zhi-Hua Zhou. Learning environmental calibration actions for policy self-evolution. In Proceedings of the 27th International Joint Conference on Artificial Intelligence (IJCAI'18), pages 3061-3067, Stockholm, Sweden, 2018.

[Zhou, 2016] Z.-H. Zhou. Learnware: On the future of machine learning. Frontiers of Computer Science, 10(4):589590, 2016. 